\title{
Scaling properties of crack branching and brittle fragmentation
}

\author{
M. Davydova ${ }^{1}$, O. Naimark ${ }^{1}$, V. Leontiev ${ }^{1}$, and S. Uvarov ${ }^{1}$ \\ ${ }^{1}$ Institute of Continuous Media Mechanics RAS, 1 Ak. Korolev str., 614013 Perm, Russia
}

\begin{abstract}
The present study is focused on the correlation of scaling properties of crack branching and brittle fragmentation with damage accumulation and a change in the fracture mechanism. The experimental results obtained from the glass fragmentation tests indicate that the size distribution of fragments has a fractal character and is described by a power law.
\end{abstract}

\section{Fragmentation of glass plates}

A comparative analysis of fragmentation statistics is carried out for quasi-static [1] and dynamic loading of glass samples. Quasi-static testing was performed in experiments with glass plates loaded in a "sandwich" to save the glass fragmentation pictures (Fig. 1a). The original photo software allowed us to determine the size and number of fragments and the total length of cracks. Two types of scaling are studied. The first type is based on the relation

$$
L(S) \sim S^{D},
$$

where $L(s)$ is the total crack length inside the square frame with a thickness $h$ (Fig. 2a), $S$ is the frame area, and $D$ is the fractal dimension. The second type is the traditional definition of the cumulative distribution of fragment sizes, in other words, the calculation of the number of fragments $N(S)$ with the size larger than $S$. The experimental results show that the size distribution of the fragments is fitted by a power law (Fig. 1b).

There exist two types of the fragmentation patterns depending on the energetic conditions leading to the breakup (Fig. 2). Figure $2 \mathrm{a}$ is a fragmentation pattern illustrating the fracture accompanied by radial crack formation. The right fragmentation pattern (Fig. 2b) has two zones corresponding to two different fracture mechanisms. The central zone has a radial crack only. In the second zone, the crack branching process is observed. Calculations of the fractal dimension using relation (1) show that for the left fragmentation pattern (Fig. 2a) the data is fitted by a single line, whereas for the right pattern the log-log representation of $L(s)$ changes a slope, and the power-law exponent $D$ decreases. A change in the fracture mechanism (from radial crack-to-crack branching) correlates with the qualitative changes in the fractal dimension.

\section{Crack branching}

A comparison of the fragmentation at quasi-static loading with the available experimental data on crack dynamics [2] suggests that there is analogy between the straight-line crack propagation at low loading levels and the radial fragmentation picture (Fig. 2a) and between the transition (under increasing load) to a new "topological" fragmentation scenario (generation of multiple tangential cracks as shown in Fig. 2b) and the branching crack dynamics.

This is an Open Access article distributed under the terms of the Creative Commons Attribution-Noncommercial License 3.0, which permits unrestricted use, distribution, and reproduction in any noncommercial medium, provided the original work is properly cited. 


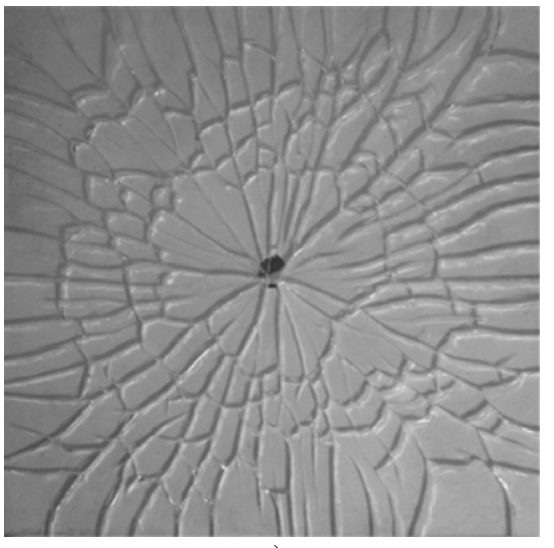

a)

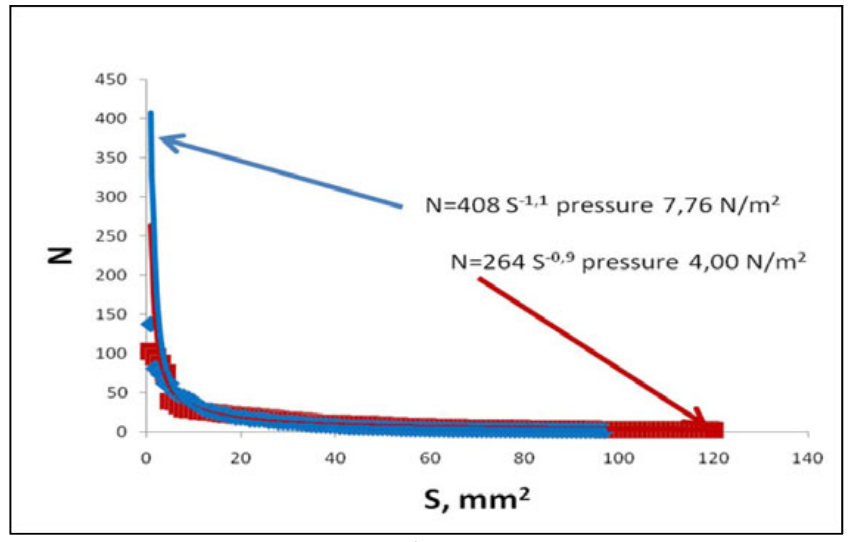

b)

Fig. 1. a) Typical fragmentation patterns. b) Cumulative distribution for two pressure values.
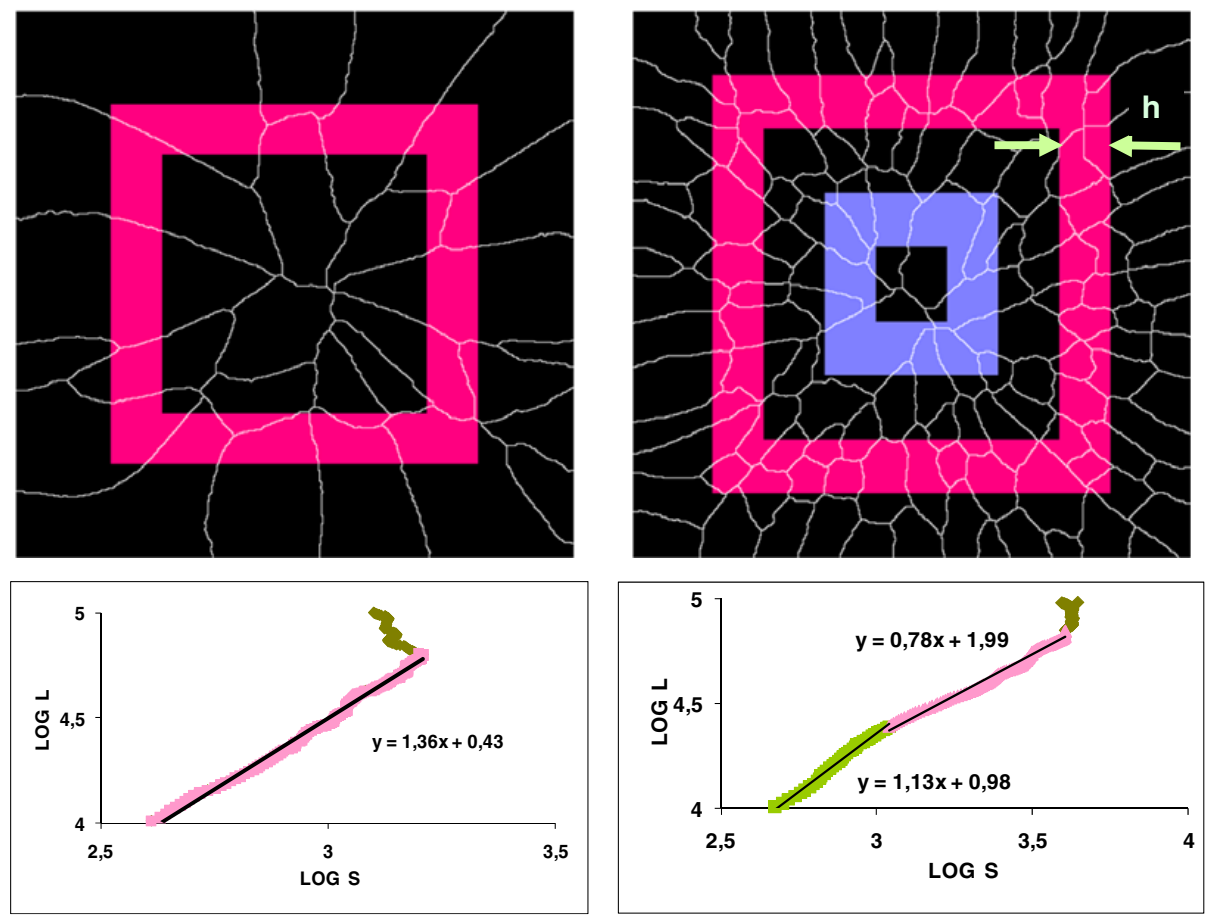

Fig. 2. Fragmentation pattern and calculation of the fractal dimension for the pressure value, a) lower than $4 *$ $10^{5} \mathrm{~N} / \mathrm{m}^{2}$ and b) higher than $4 * 10^{5} \mathrm{~N} / \mathrm{m}^{2}$.

The confirmation of the fact that the fractal characteristic correlates with the fracture mechanism was observed in experiments on dynamics crack propagation in PMMA. The experiments revealed two regimes of crack propagation: steady-state or single crack propagation and branching. In the steadystate mode, the crack propagates at a low speed (up to $300 \mathrm{~m} / \mathrm{s}$ or $0.2 \mathrm{~V}_{\mathrm{R}}$ ) and produces the smooth fracture surface. In the branching mode, the crack speed is higher and the crack surface is rougher. We defined the roughness exponent of PMMA fracture surfaces in order to get the quantitative confirmation of the assumption that the crack surface roughness depends on the crack velocity. The roughness exponent $H$ can be defined from the equation $Z_{\max }(r) \sim r^{H}$, where $z$ is the height of the surface profile, 


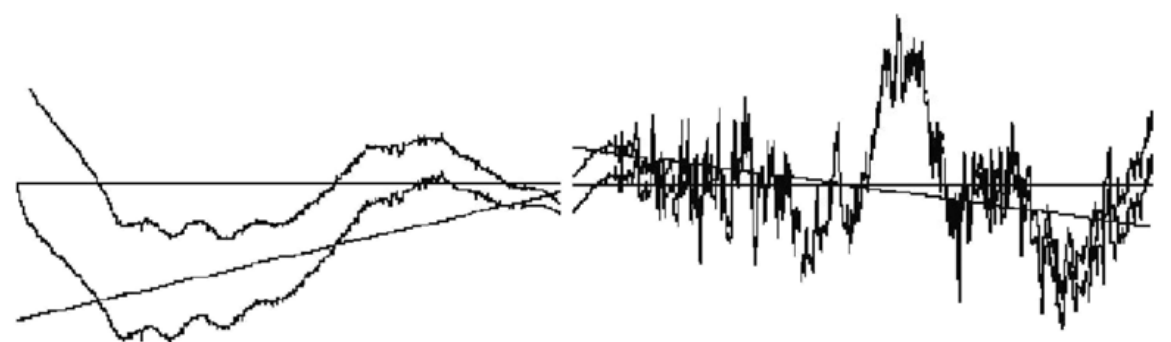

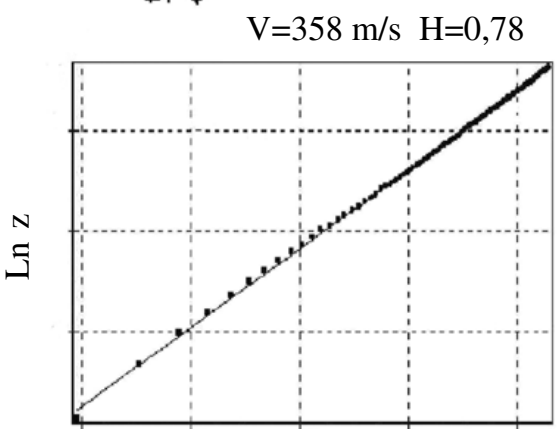

$\operatorname{Ln}(r)$

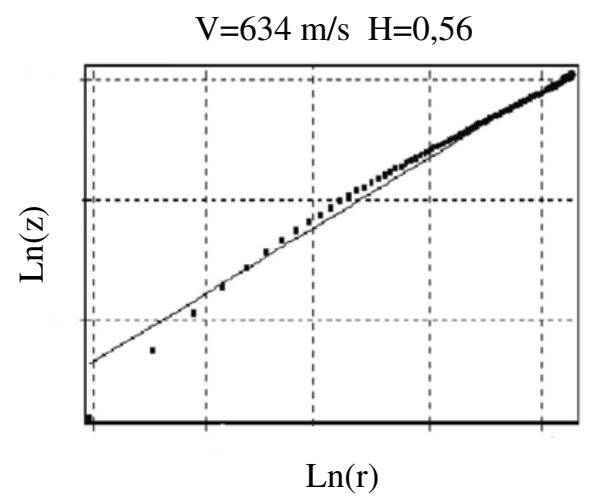

b)

Fig. 3. Surface profile and definition of the Hurst exponent for crack spreading at velocities: a) $538 \mathrm{~m} / \mathrm{s}$, b) $634 \mathrm{~m} / \mathrm{s}$.

and $r$ is the coordinate within the horizontal plane. The results of statistical data processing demonstrate that the surface roughness increases (the roughness exponent decreases) with the crack velocity growth (Fig. 3). In other words, a change in the fracture regime from single crack propagation to branching leads to a decrease in the roughness exponent.

\section{Fragmentation of glass rods}

The fragmentation statistics was studied in recovery dynamic experiments with loaded quartz cylindrical rods using a ballistic set-up (a gas gun with a bore diameter of $19.3 \mathrm{~mm}$ and a velocity registration system). The sectional glass rod was composed of the transmitting and main parts covered by an elastic shell. The transmitting part was used for realization of uniaxial loading produced by a cylindrical projectile of mass $13.9 \mathrm{~g}$ accelerated up to the velocities of 6-20 m/s. The mass of the fragments passing through sieves was obtained by weighting the fragments using an electronic balance HR-202i (accuracy $10^{-4} \mathrm{~g}$ ). The mass of the fragments corresponding to the maximum of the probability density function varied in the range from $2 * 10^{-4} \mathrm{~g}$ to $6 * 10^{-4} \mathrm{~g}$. The cumulative fragment size distribution, the number of fragments $N(m)$ with the mass greater than a specified value $m$ were fitted by a power law.

The ballistic set-up was modified to avoid the possible influence of the reflected wave on the fragmentation scenario. The sample was placed into a steel cylinder filled with plastic foam. The sectional glass rod was composed of transmitting, main and outer parts. The fragmentation statistics was analyzed by varying the sample size and load intensity (projectile velocity). The results of the experiments indicate that the variation in the sample size and loading conditions does not lead to the change in the probability density function and cumulative mass distribution. The mass of the fragments corresponding to the maximum of the probability density function is independent of the projectile energy.

Finally, cumulative distributions illustrating the relation between the numbers of fragments and their linear dimension are represented as a log-log plot (Fig. 4). The linear dimension is defined as a 
EPJ Web of Conferences

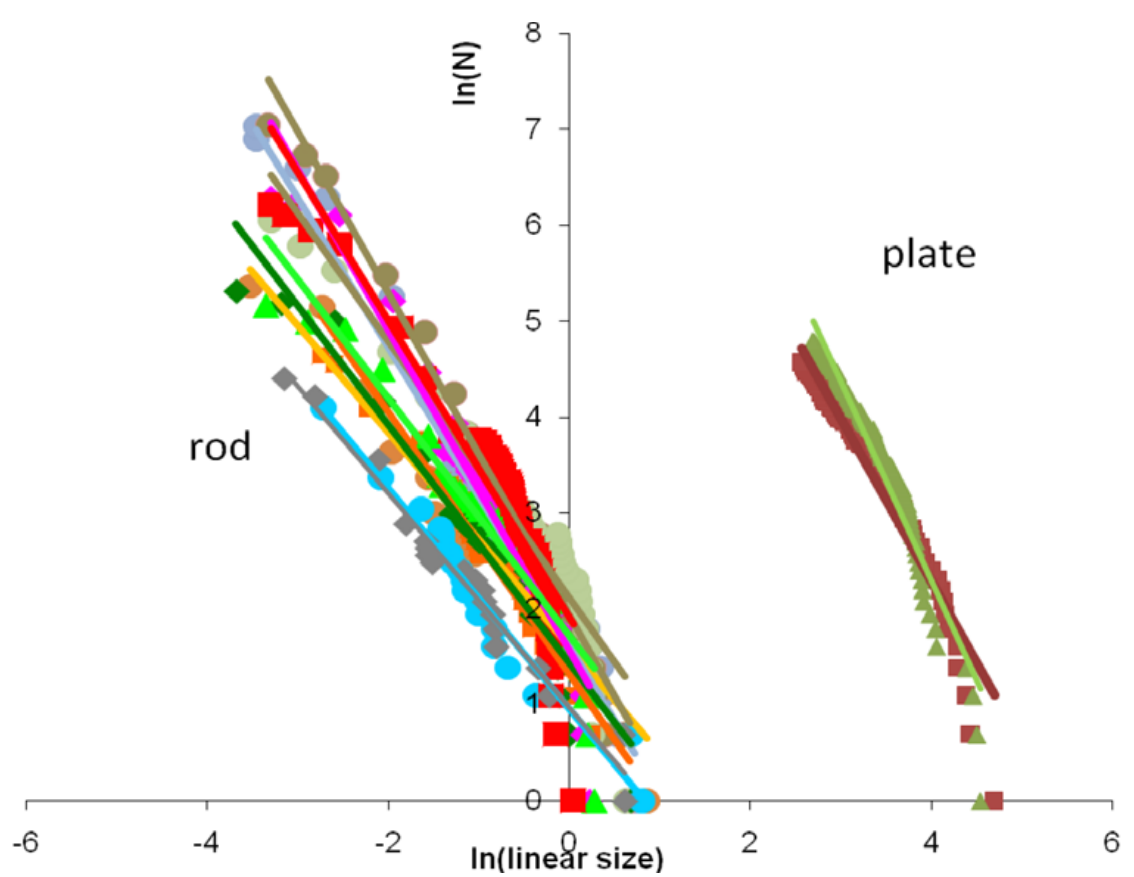

Fig. 4. Double logarithmic plot of the cumulative distribution function for a plate and a rod.

cube root of mass or a square root of area. The distribution is fractal by nature with a power law in the form $N(>r)=C r^{-D}$, where $N$ is the number of fragments with a characteristic linear dimension greater than $r$. The fractal dimension $D$ varies from 1.6 to 2.0 for a plate and from 1.1 to 1.7 for a rod.

\section{Conclusions}

The glass fragmentation experiments have shown that:

- The fragmentation patterns of glass plates are fractal by nature.

- The variation in the fracture mechanism of plates correlates with the changes in the fractal dimension.

- The cumulative mass distribution of the fragments for 2D and 3D samples is described by a power law.

- The statistical number-size distribution for the observed type of fragmentation has a fractal character $N(>r)=C r^{-D}$, where $N$ is the number of objects with characteristic linear dimensions greater than $r$.

The author would like to acknowledge the Russian Foundation for Basic Research (grant RFBR 08-01-00699, grant RFBR 09-08-99097, grant RFBR 09-01-92005, grant RFBR 09-01-92441).

\section{References}

1. M. Davydova and D. Davydov, Materials Science Forum, 567-568, 289, (2007)

2. O.B. Naimark, in: Advances in Multifield Theories of Continua with Substructure (Birkhauser Volume Edition, Boston) 75, (2004) 